\title{
Effect of structural deformation on carrier accumulation in semiconducting carbon nanotubes under an external electric field
}

\author{
Akiko Hasegawa and Susumu Okada* \\ Graduate School of Pure and Applied Sciences, University of Tsukuba, Tsukuba, Ibaraki 305-8571, Japan
}

\begin{abstract}
We study the effect of structural deformation on carrier accumulation in semiconducting carbon nanotubes (CNTs) under the external electric field, on the basis of fthe density functional theory combined with the effective screening medium method. The capacitances of the CNTs with ellipsoidal and squashed cross sections are different from that of the pristine CNT owing to the distribution of accumulated carriers depending on the CNT deformation and arrangements with respect to the electric field direction. The results suggest that the cross section and arrangement of deformed CNTs result in gate voltage variations for both electron and hole injection, causing the degradation of CNT-based field-effect electronic devices.
\end{abstract}

\section{Introduction}

Ever since the discovery of the nanometer scale tubular form of the rolled-up graphene, ${ }^{1)}$ i.e. carbon nanotubes (CNTs), the CNTs have attracted much attention owing to their unique structural and electronic properties that are applicable to wide areas of the current and future nanotechnology. CNTs are either metals or semiconductors depending on the discretization condition on the metallic electronic energy band of graphene sheet determined by their atomic arrangements along the circumference and diameters. $^{2-4)}$ Furthermore, owing to the strong covalent $\mathrm{sp}^{2}$ network of $\mathrm{C}$ atoms, CNTs possess remarkable mechanical strength, ${ }^{5,6)}$ resulting in high thermal conductivity. ${ }^{7-9)}$ In addition to these intrinsic properties, defects, edges, and structural deformation lead to further variations in their physical properties. ${ }^{10-20)}$ CNTs possess spin polarization around atomic defects due to unsaturated $\sigma$ bonds and nonbonding $\pi$ states. ${ }^{12}$ ) The nanometer-scale tubular space of CNTs can contain foreign atoms and molecules resulting in further functions and properties due to the substantial interaction between host CNT and guest materials. ${ }^{21-23)}$ These characteristic features of CNTs make them a premier material for wide areas of nanotechnology. For instance, it has been experimentally reported that CNTs work as a conducting channel of field-effect transistors

*E-mail: sokada@comas.frsc.tsukuba.ac.jp 
(FETs). ${ }^{24-28)}$

In the FET structures, CNTs intrinsically possess defects and structural deformations that seriously modulate the electronic structure of CNTs as stated above. For CNTs with atomic and topological defects in FET structures, the carrier accumulation strongly depends on the mutual orientation of the defects with respect to the applied external electric field by the electron polarization around the defects. ${ }^{29,30)}$ In addition to the defects, structural deformations are also induced by forming hybrid structures with foreign materials, such as other CNTs, insulating substrates, and metal electrodes. ${ }^{16-20)}$ In such situations, CNTs possess ellipsoidal and squashed cross sections due to the substantial interaction with foreign materials. Although several researches have investigated the electronic properties of CNTs under an external electric field, ${ }^{31-36)}$ the fundamental properties of carrier accumulation into CNTs with FET structures by an electric field are not fully understood. In particular, it is still unclear how the structural deformations of CNTs affect the electronic properties and charge accumulation under an electric field, which is an important issue for the application of CNTs in semiconducting electronic devices.

In this manuscript, we aim to elucidate the fundamental electronic properties of zigzag (20,0)CNTs with ellipsoidal and squashed cross sections under an electric field based on first-principles total-energy calculations using density functional theory. The calculations showed that the capacitances of deformed CNTs strongly depend on their shapes and relative arrangement to the electrode. Furthermore, accumulated carriers in and field concentration around the CNTs also depend on the CNT cross section and mutual arrangement to the electrode. The results give theoretical guiding principles for designing CNT-FET device structures in terms of carrier accumulation.

\section{Method and models}

All of the calculations were performed using the density functional theory (DFT) ${ }^{37,38)}$ with a Simulation Tool for Atom TEchnology (STATE) package. ${ }^{39}$ ) To express the exchange correlation potential among the interacting electrons, the local density approximation is applied with the Perdew-Zunger functional form fitting to the Quantum Monte Carlo results on the the homogeneous electron gas. ${ }^{40,41)}$ We use an ultrasoft pseudopotential to describe the interactions between the valence electrons and the ions generated by the Vanderbilt scheme. ${ }^{42)}$ The valence wave functions and charge density were expanded with a plane-wave basis set with cutoff energies of 25 and 225 Ry, 
(a)

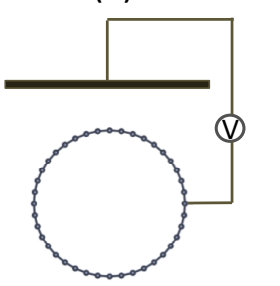

(b)

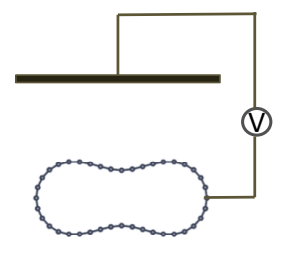

(c)

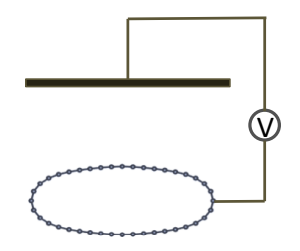

(d)

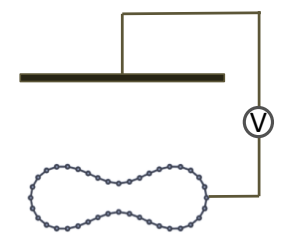

(e)

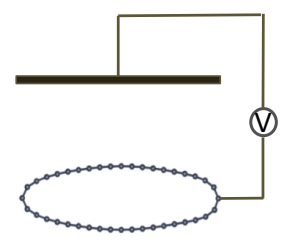

Fig. 1. Structural models of deformed CNT under an electric field. The (20,0) CNTs with (a) circular, (b) moderately squashed (MS), (c) moderately ellipsoidal (ME), (d) tightly squashed (TS), and (e) tightly ellipsoidal (TE) cross sections are arranged below the planar electrode denoted by black thick lines.

respectively. To perform the Brillouin zone integration, six equidistant $k$-points were sampled along the direction of the CNT.

The effective screening medium method is applied to investigate the electronic properties of the CNTs with structural deformations under an electric field within DFT. ${ }^{43}$ ) In this work, $(20,0)$ CNTs were considered with squashed and ellipsoidal cross sections as the deformed CNTs. To simulate the CNT-FET device structure, we consider the structural model shown in Fig. 1, in which the CNT is located below the planar gate electrode simulated by an effective screening medium with the infinite permittivity to mimic an ideal metal electrode. The center of the deformed CNTs is located at 15.88 $\AA$ below the electrode. In addition, we consider the other CNT arrangements in which the deformed CNTs possess the same spacing between the electrode and the CNT wall of 3.18 and $6.28 \AA$. An electric field is applied between the electrode and the CNT up to the gate voltage, which injected 0.5 carriers into CNTs per unit cell. The CNTs with squashed and ellipsoidal shapes were obtained by performing the structural optimization on $(20,0)$ CNTs under zero field with a structural constraint that the topmost and the bottommost dimer rows are fixed. The lattice parameter along the tube axis is fixed at $4.26 \AA$ corresponding to the single periodicity of zigzag CNTs. To investigate the electronic properties of an isolated CNT, each CNT is separated by an $8 \AA$ interwall spacing from its periodic images. During the calculations under the conditions with a finite electric field, the atomic geometries were kept the same as those with a zero electric field. 
(a)

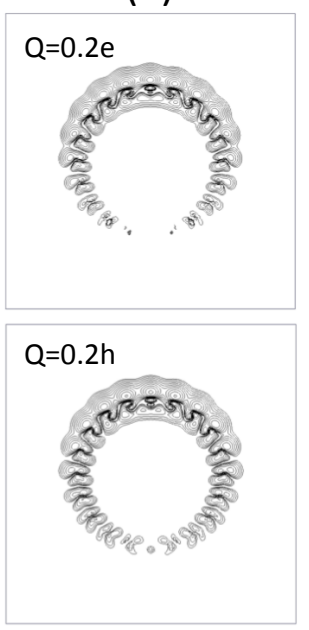

(b)

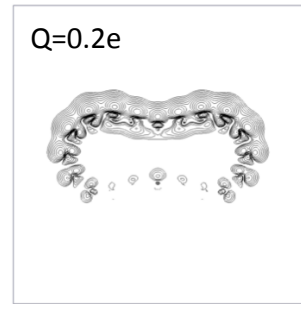

$Q=0.2 h$

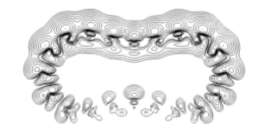

(c)

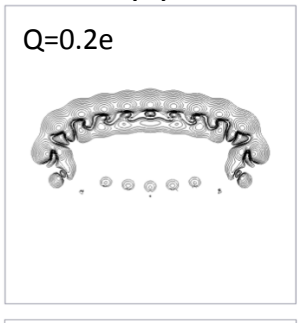

$Q=0.2 h$

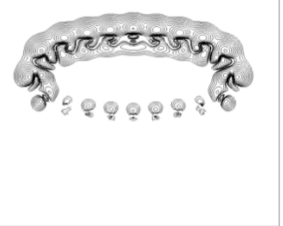

(d)
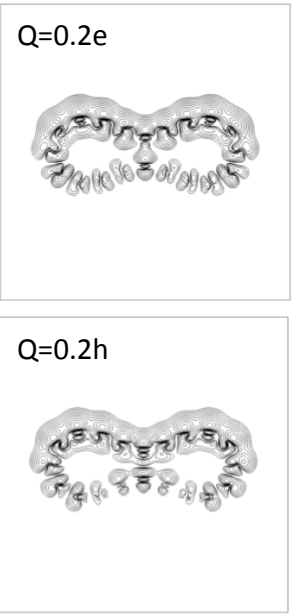

(e)

$\mathrm{Q}=0.2 \mathrm{e}$

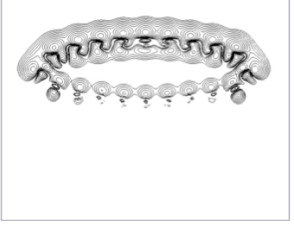

$Q=0.2 h$

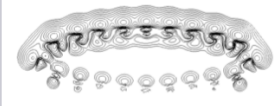

Fig. 2. Contour plots of accumulated carriers by the electric field, $\Delta n(\boldsymbol{r})$, of $(20,0)$ CNT with (a) circular, (b) moderately squashed (MS), (c) moderately ellipsoidal (ME), (d) tightly squashed (TS), and (e) tightly ellipsoidal (TE) cross sections. In each panel, $0.2 \mathrm{e}$ and $0.2 \mathrm{~h}$ denote 0.2 electrons and 0.2 holes per unit cell, respectively.

\section{Results and discussion}

Figure 2 shows the distributions of accumulated electrons (0.2e/cell) and holes $(0.2 \mathrm{~h} /$ cell $)$ in CNTs with circular, squashed, and ellipsoidal cross sections by the external electric field. In this case, the center of the CNT is located at $15.88 \AA$ below the electrode. For all CNTs, the accumulated carriers are primarily distributed on the outer part of the CNT wall at the electrode side for both electron and hole accumulations. In addition to the main distribution, the holes and electrons are also slightly induced on the inner wall of the CNTs at the electrode side under positive and negative bias voltages, respectively. Furthermore, for the case of CNTs with squashed and ellipsoidal shapes, electrons and holes are induced in the inner and outer walls, respectively, at the opposite side to the electrode. In accordance with the carrier distribution induced by the electric field, the hybrid system consisting of the deformed CNT and electrode can be regarded as two capacitors connected in series. In contrast to the CNTs with structural deformations, charge redistribution in the pristine CNT induced by the electric field exhibits different natures: The accumulated/depleted carriers oscillate along their circumference.

Figure 3 shows the contour plots of electrostatic potential and the vector plots of corresponding electric field under the electron $(0.2 \mathrm{e} /$ cell $)$ and hole $(0.2 \mathrm{~h} /$ cell $)$ accumulations. The potential gradient or the electric field is primary concentrated at the 
(a)

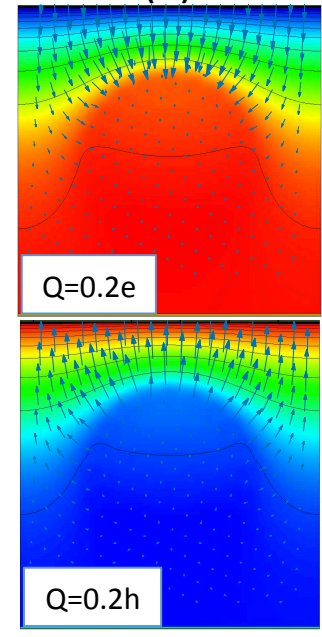

(b)

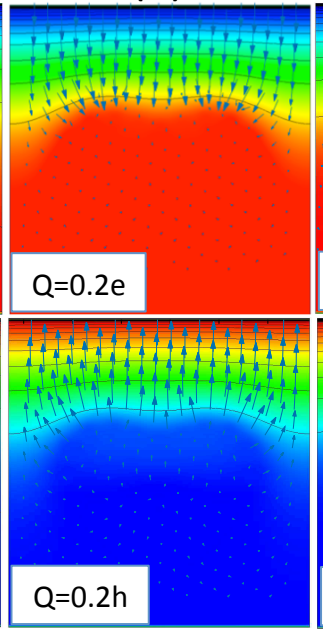

(c)

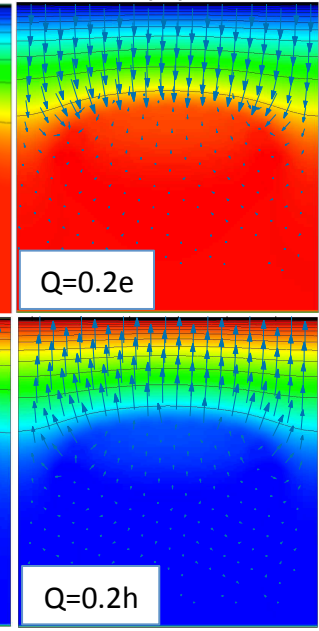

(d)
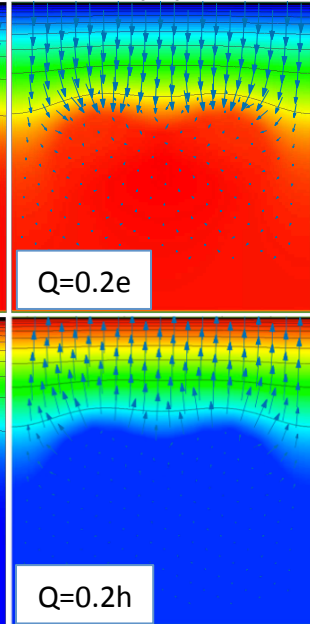

(e)

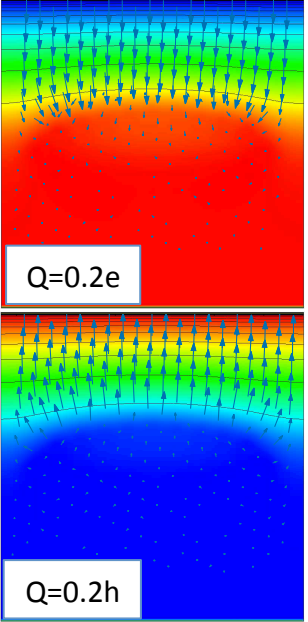

Fig. 3. (Color online) Contour and vector plots of electrostatic potential and electric field, respectively, of (20,0)CNT with (a) circular, (b) moderately squashed (MS), (c) moderately ellipsoidal (ME), (d) tightly squashed (TS), and (e) tightly ellipsoidal (TE) cross sections. In each panel, $0.2 \mathrm{e}$ and $0.2 \mathrm{~h}$ denote 0.2 electrons and 0.2 holes per unit cell, respectively.

electrode side of the CNTs, while the electric field is absent around the opposite side of the CNTs, because of the concentration of the accumulated carriers at the electrode side of the CNTs. On the other hand, the detailed electrostatic potential and electric field strongly depend on the shape of CNTs: For the pristine and squashed CNTs, the field concentration occurs around the part of the CNT wall with a large curvature as in the case of the conducting materials with a protruding portion in the classical electrodynamics. In contrast, for the CNTs with the ellipsoidal cross section, the almost constant field emerges around the outer wall of the CNTs, since the CNTs possess a flat region with respect to the electrode.

Figure 4 shows the calculated total capacitance of CNTs,

$$
C=\frac{d Q}{d \mu}=\frac{C_{0} D}{C_{0}+D}
$$

where $C_{0}$ and $D$ denote the geometrical capacitance and density of states of CNT, respectively, ${ }^{36)}$ as a function of the gate voltage. The capacitance strongly depends on both the gate voltage and CNT cross sections. The pristine CNT possesses the largest capacitance among the five CNTs studied here. The largest capacitance of the pristine CNT is ascribed to the structural model studied here, that is the center of the CNT is located at $15.88 \AA$ below the electrode. Thus, the spacing between the electrode and the wall of the pristine CNT is smaller than that of the other deformed CNTs, 
(a)

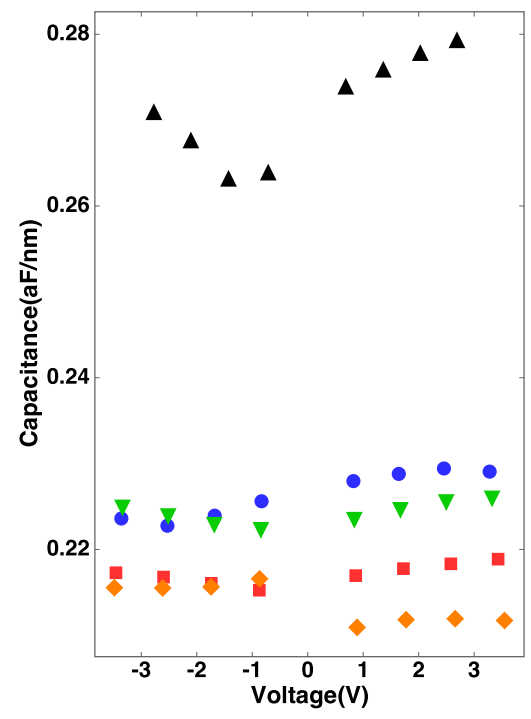

(b)

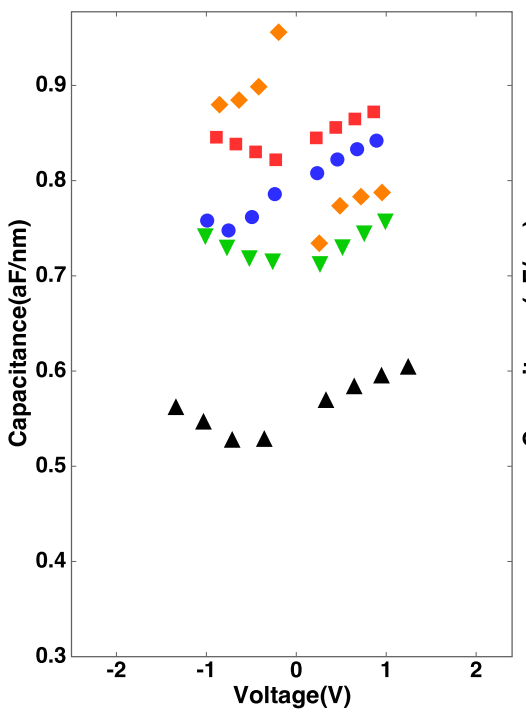

(c)

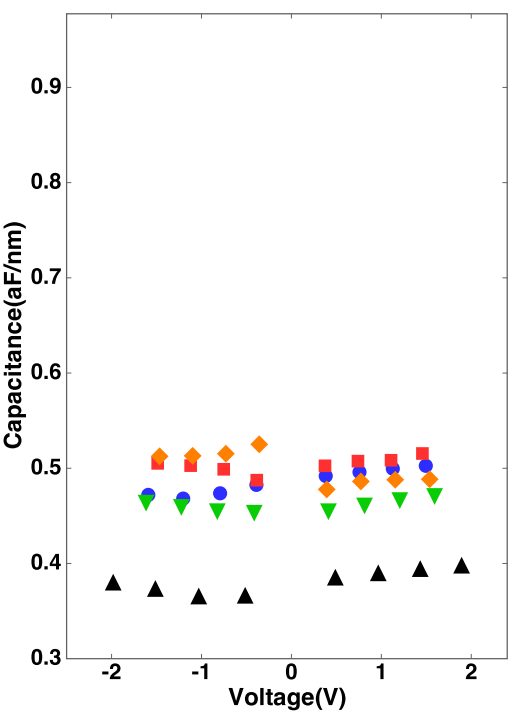

Fig. 4. (Color online) (a) Capacitances of pristine and deformed (20,0)CNTs situated below the electrode by the spacing of $15.88 \AA$ between the center of CNTs and the electrode. Capacitance of pristine and deformed $(20,0) \mathrm{CNTs}$ arranged with the spacing between the electrode and the CNT walls of (b) 3.18 and (c) $5.29 \AA$ A. Triangles, circles, inverse triangles, squares, and rhombuses denote capacitances of CNTs with circular, moderately squashed (MS), moderately ellipsoidal (ME), tightly squashed (TS), and tightly ellipsoidal (TE) cross sections, respectively.

leading to the largest geometrical (classical) capacitance $C_{0}$. Indeed, the capacitance decreases with increasing structural deformation of CNTs causing the increase in the spacing between the CNT and the electrode. For instance, the moderately ellipsoidal and squashed CNTs possess a smaller capacitance than that with the tightly deformed cross sections. In addition to the spacing between the electrode and the CNT wall, the cross section of CNTs also affects the capacitance. For the deformed CNT with the same length in its minor axis, the capacitance of the squashed CNT is larger than that of the ellipsoidal CNT. In the case of the CNT with the squashed cross section, the two convex regions of the wall cause a larger capacitance than that of the CNT with an ellipsoidal cross section.

To check the effect of the structural deformation on the capacitances, we calculate the capacitance of pristine and deformed CNTs possessing the same spacing between the electrode and the CNT wall of 3.18 and $6.28 \AA$. Figures 4(b) and 4(c) show the capacitances under the spacing of 3.18 and $6.28 \AA$, respectively. The capacitance of all CNTs are larger under the spacing of 3.18 Athan under the spacing of $6.28 \AA$. In addition to the spacing, the capacitance increases with increasing deformation, which causes the 
(a)

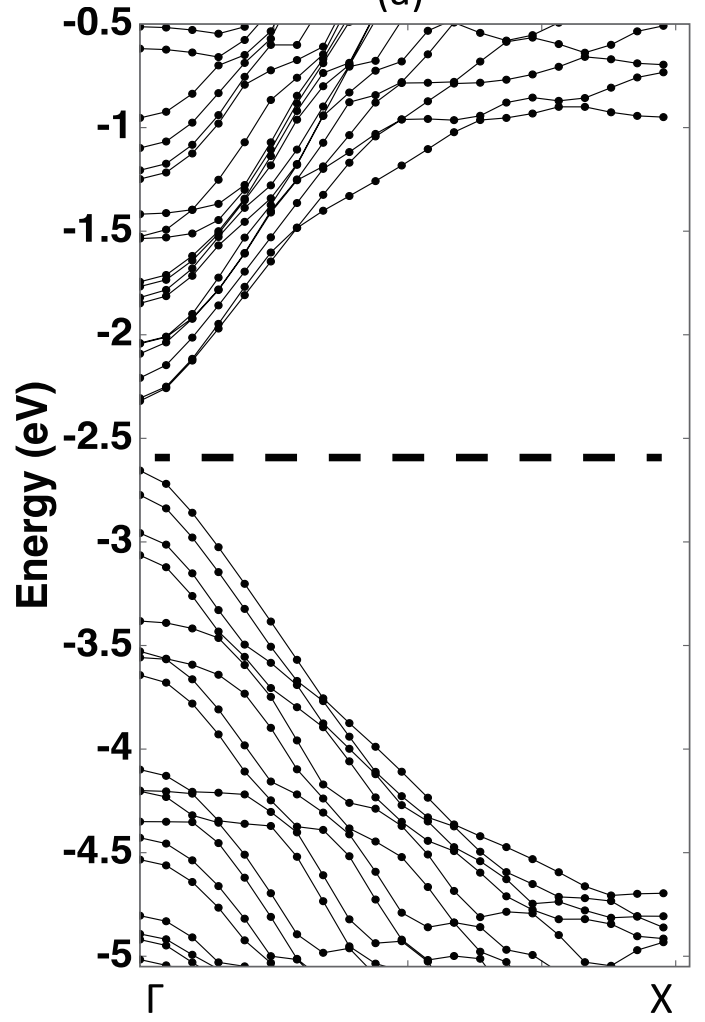

(b)

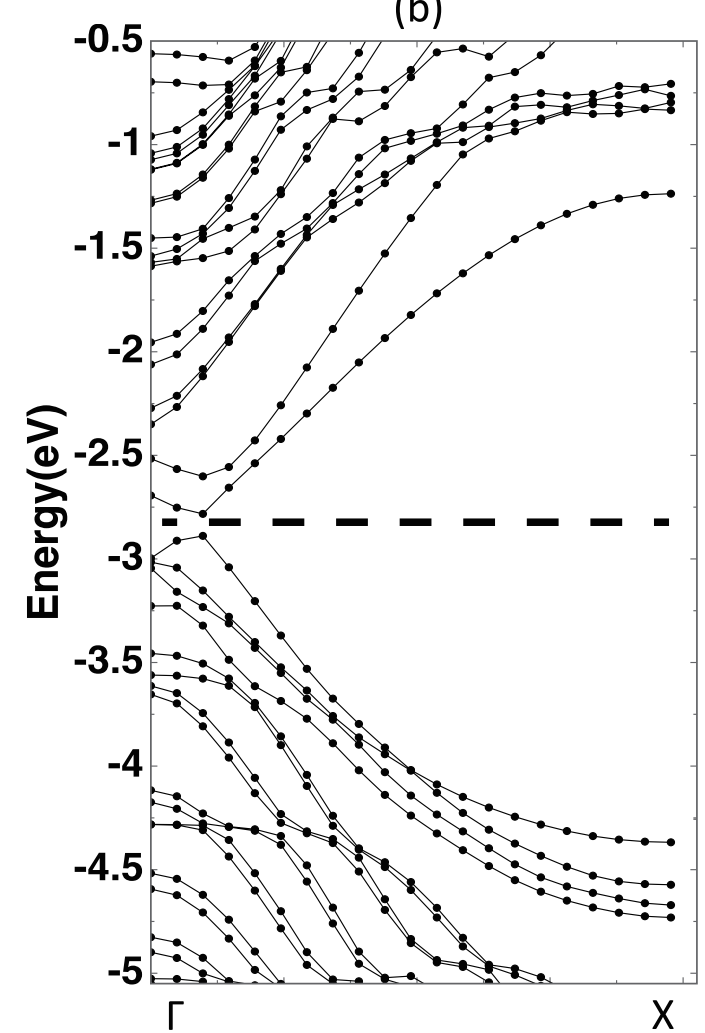

Fig. 5. Electronic energy bands of (a) CNT(TE) and CNT(TS). Horizontal dotted lines denote the Fermi level energy.

increases in the lateral area of the deformed CNT with respect to the gate electrode. Indeed, under the fixed CNT-electrode spacing, the CNT with a tightly squashed shape possesses the largest capacitance. Thus, the results indicate that the deformation of the CNT sensitively affects its capacitance in the FET structure.

In addition to the structural dependence of the capacitances, the capacitance of CNTs also exhibit the gate voltage dependence for all cases, due to the quantum effect arising from the electronic structure of the CNTs. For all cases except the CNT with the squashed shape [CNT(TS)], the capacitance increases with increasing positive and negative gate voltages, because of the increase in the number of electron states for corresponding gate voltage. In the case of the CNT(TS), the capacitance substantially increases by applying the negative gate voltage. This unusual feature in the capacitance is ascribed to the electronic structure of the CNT(TS). As shown in Fig. 5, the CNT(TS) is a metal in which the bonding and antibonding $\pi$ states cross the Fermi level near the 
$\Gamma$ point due to the substantial structural deformation, although the other CNTs retain semiconducting electronic structures. Owing to the metallic nature of the CNT(TS), the electron states below the Fermi level cause the large capacitance in the negative gate voltage, since the total capacitance increases with increasing number of electron states.

It is worth investigating how the distribution of accumulated carriers and electric field depend on the relative arrangement of the deformed CNT to the electric field. Figure 6 shows the contour and vector plots of the accumulated carrier and electric field, respectively, of the deformed CNTs tilted to the electric field under $0.2 \mathrm{e} /$ cell and $0.2 \mathrm{~h} /$ cell injections. For all CNTs with the parallel arrangement to the electric field ( $\theta=90$ deg.), accumulated electrons are mainly concentrated at the top of the CNT facing to the electrode. Furthermore, accumulated/depleted carriers oscillate along the circumference as in the case of the CNT with the circular cross section. Owing to the large curvature of the CNTs with squashed and ellipsoidal cross sections with respect to the electrode, the electric field highly concentrates around the protruding region. With decreasing the CNT orientation to the field, the charge oscillation and field concentration decrease.

Since the rotation of the CNT modulates the distribution of accumulated/depleted carriers by the electric field, the capacitances of the CNTs with squashed and ellipsoidal cross sections may depend on the relative arrangement to the electric field. Figure 7 shows the calculated capacitances of the deformed CNTs with the rotational angles of 30,60 , and $90^{\circ}$ with respect to the electric field under the fix distance of $15.88 \AA$ between the electrode and the center of the CNTs. The capacitance strongly depends on their mutual arrangement to the electric field. By increasing the rotational angle, the capacitances of the deformed CNTs monotonically increase. The capacitance is determined by the competition between the decrease in the vacuum spacing and the increase in the area of the CNT with respect to the electrode. Therefore, the CNT orientation with respect to the electric field also affects the carrier accumulation of the CNT with structural deformations in FET structures.

\section{Summary}

The electronic structure of CNTs with squashed and ellipsoidal cross sections was studied under a vertical electric field in terms of the CNT cross sections and their mutual arrangement relative to the gate electrode based on DFT combined with the effective 


\section{(a)}
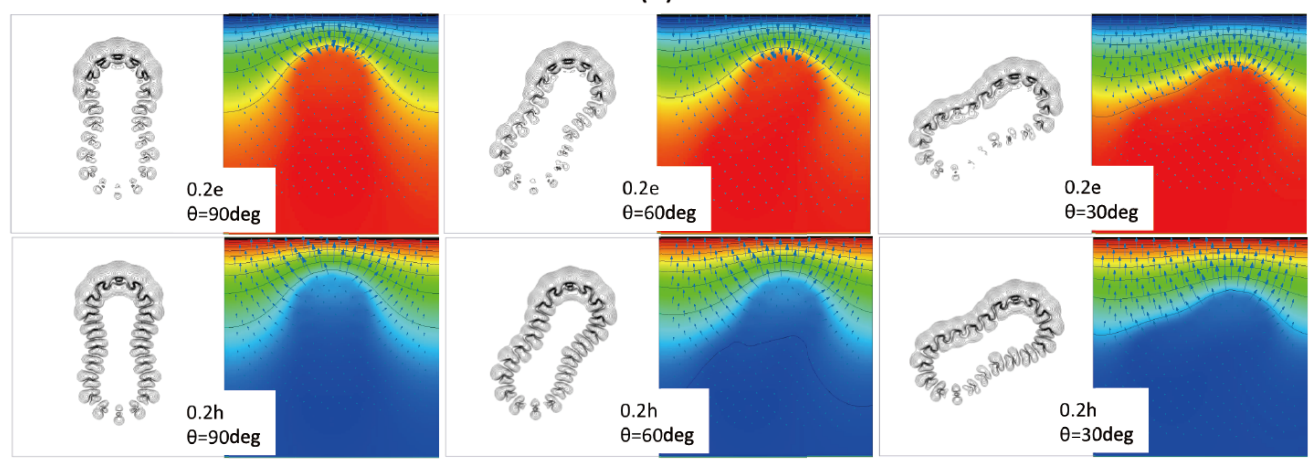

(b)
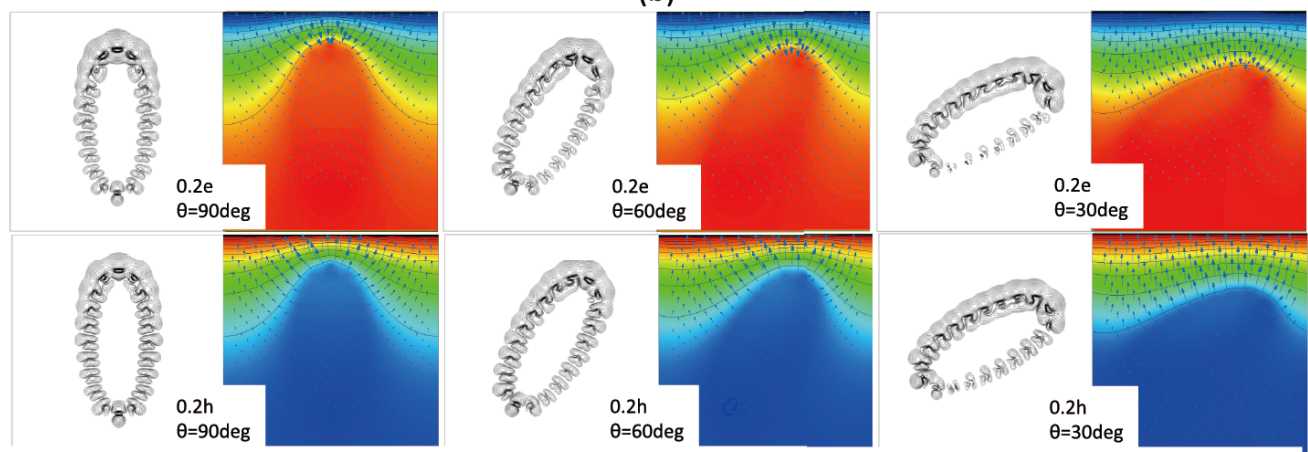

(c)
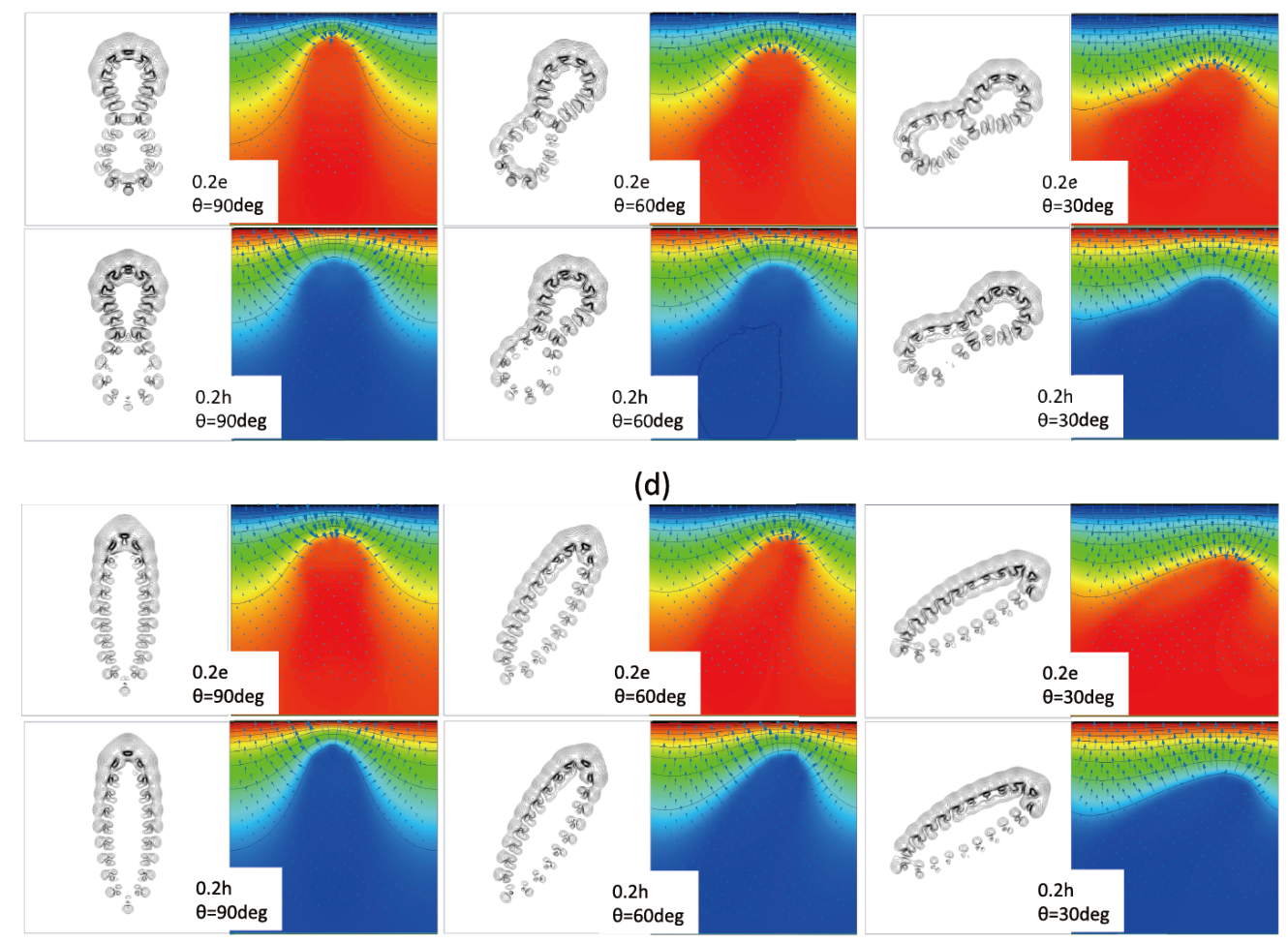

Fig. 6. (Color online) Contour and vector plots of accumulated carriers and electric field, respectively, of (20,0)CNT with (a) moderately squashed (MS), (b) moderately ellipsoidal (ME), (c) tightly squashed (TS), and (d) tightly ellipsoidal (TE) cross sections under the rotational angles of $\theta=30,60$, and $90^{\circ}$ with respect to the electric field under the carrier injection of 0.2 electrons and 0.2 holes per unit cell. 
(a)

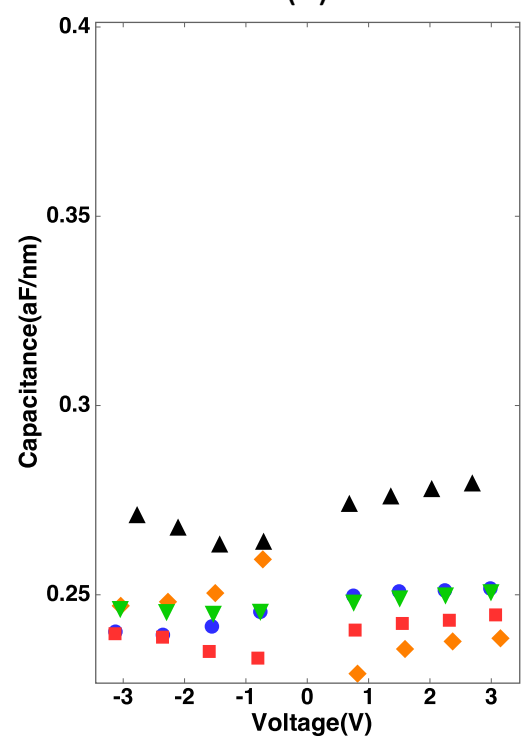

(b)

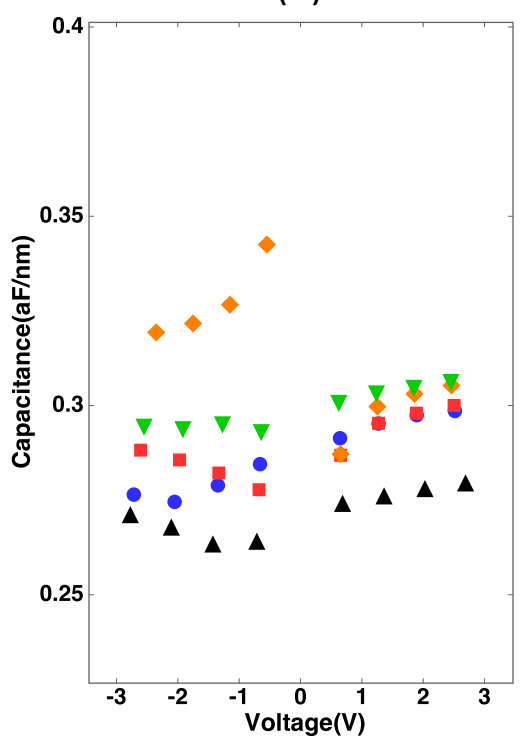

(c)

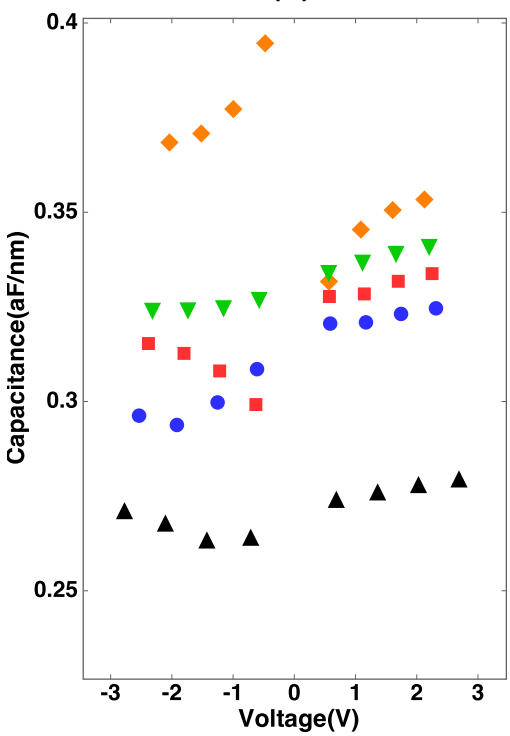

Fig. 7. (Color online) Capacitances of pristine and deformed (20,0)CNTs with the rotational angles of (a) 30, (b) 60, and (c) $90^{\circ}$. Triangles, circles, inverse triangles, squares, and rhombuses denote capacitances of CNTs with circular, moderately squashed (MS), moderately ellipsoidal (ME), tightly squashed (TS), and tightly ellipsoidal (TE) cross sections, respectively.

screening medium method. Calculations showed that the carrier accumulation due to the external electric field strongly depends on the cross section of CNTs: Electrons and holes are primarily induced at the outside of the wall at the electrode side. In addition, the carriers oscillate along the field for the CNT with the circular cross sections, while the carriers oscillate along the field for the CNTs with squashed and ellipsoidal cross section. The field concentration occurs at the protruding areas of the CNTs with the squashed cross section. In sharp contrast, owing to the flat nature of the wall to the electrode, CNTs with the ellipsoidal cross section do not exhibit field concentration outside the wall. Furthermore, the carrier accumulation in CNTs with structural deformation also strongly depends on the relative direction of CNTs to the electric field. According to the characteristic distribution of accumulated carriers, the capacitances of the CNTs strongly depend on their cross section and relative arrangement to the electrode. Therefore, the structural deformation and their relative arrangement to the field are important factors when fabricating CNT electronic devices.

\section{Acknowledgements}

This work was supported in part by a Grant-in-Aid for Scientific Research from the Ministry of Education, Culture, Sports, Science and Technology of Japan and the Joint 
Research Program on Zero-Emission Energy Research, Institute of Advanced Energy, Kyoto University. Computations were performed on a NEC SX-8/4B at the University of Tsukuba, a SGI ICE XA/UV at the Institute for Solid State Physics, The University of Tokyo, and a NEC SX-Ace at the Cybermedia Center, Osaka University. 


\section{References}

1) S. Iijima, Nature 354, 56 (1991).

2) N. Hamada, S.-I. Sawada, and A. Oshiyama, Phys. Rev. Lett. 68, 1579 (1992).

3) R. Saito, M. Fujita, M. S. Dresselhaus, and G. Dresselhaus, Appl. Phys. Lett. 60, 2204 (1992).

4) K. Tanaka, K. Okahara, M. Okada, and T. Yamabe, Chem. Phys. Lett. 191, 469 (1992).

5) R. S. Ruoff and D. C. Lorents, Carbon 33, 925 (1995).

6) M. M. J. Treacy, T. W. Ebbesen, and J. M. Gibson, Nature 381, 678 (1996).

7) S. Berber, Y.-K. Kwon, and D. Tománek, Phys. Rev. Lett. 84, 4613 (2000).

8) T. Yamamoto, S. Watanabe, and K. Watanabe, Phys. Rev. Lett. 92, 075502 (2004).

9) T. Yamamoto and K. Watanabe, Phys. Rev. Lett. 96, 255503 (2006).

10) M. Igami, T. Nakanishi, and T. Ando, J. Phys. Soc. Jpn. 68, 716 (1999).

11) P. O. Lehtinen, A. S. Foster, Y. Ma, A. V. Krasheninnikov, and R. M. Nieminen, Phys. Rev. Lett. 93, 187202 (2004).

12) Y. Ma, P. O. Lehtinen, A. S. Foster, and R. M. Nieminen, New J. Phys. 6, 68 (2004).

13) S. Lee, G. Kim, H. Kim, B.-Y. Choi, J. Lee, B. W. Jeong, J. Ihm. Y. Kuk, and S.-J. Kahng, Phys. Rev. Lett. 95, 166402 (2005).

14) S. Okada, K. Nakada, K. Kuwabara, K. Daigoku, and T. Kawai, Phys. Rev. B 74, 121412(R) (2006).

15) S. Okada and A. Oshiyama, J. Phys. Soc. Jpn. 72, 1510 (2003).

16) M. S. C. Mazzoni and H. Chacham, Appl. Phys. Lett. 76, 1561 (2000).

17) N. G. Chopra, L. X. Benedict, V. H. Crespi, M. L. Cohen, S. G. Louie, and A. Zettel, Nature 377, 135 (1995).

18) H. Mehrez, A. Svizhenko, M. P. Anantram, M. Elstner, and T. Frauenheim, Phys. Rev. B 71, 155421 (2005).

19) J.-Q. Lu, J. Wu, W. Duan, F. Liu, B.-F. Zhu, and B.-L. Gu, Phys. Rev. Lett. 90, 156601 (2003).

20) C. Gómez-Navarro, J. J. Sáenz, and J. Gómez-Herrero, Phys. Rev. Lett. 96, 076803 (2006).

21) B. W. Smith, M. Monthioux, and D. E. Luzzi, Nature 396, 323 (1998). 
22) K. Hirahara, K. Suenaga, S. Bandow, H. Kato, T. Okazaki, H. Shinohara, and S. Iijima, Phys. Rev. Lett. 85, 5384 (2000).

23) S. Okada, S. Saito, and A. Oshiyama, Phys. Rev. Lett. 86, 3835 (2001).

24) S. J. Tans, A. R. M. Verschueren, and C. Dekker, Nature 393, 49 (1998).

25) R. Martel, T. Schmidt, H. R. Shea, T. Hartel, and Ph. Avouris, Appl. Phys. Lett. 73, 2447 (1998).

26) Y. Nosho, Y. Ohno, S. Kishimoto, and T. Mizutani, Appl. Phys. Lett. 86, 073105 (2005).

27) Y. Nosho, Y. Ohno, S. Kishimoto, and T. Mizutani, Nanotechnology 17, 3412 (2006).

28) D.-M. Sun, M. Y. Timmermans, Y. Tian, A. G. Nasibulin, E. I. Kauppinen, S. Kishimoto, T. Mizutani, and Y. Ohno, Nat. Nanotechnol. 6, 156 (2011).

29) U Ishiyama, N.-T. Cuong, and S. Okada, Jpn. J. Appl. Phys. 53, 115102 (2014).

30) U Ishiyama, N.-T. Cuong, and S. Okada, Jpn. J. Appl. Phys. 54, 065101 (2015).

31) K. Tada and K. Watanabe, Jpn. J. Appl. Phys. 39, 268 (2000).

32) C. Kim and B. Kim, Phys. Rev. B 65, 165418 (2002).

33) H. Zhang and Y. Miyamoto, Appl. Phys. Lett. 95, 053109 (2009).

34) A. Yamanaka and S. Okada, Appl. Phys. Express 5, 095101 (2012).

35) A. Yamanaka and S. Okada, Appl. Phys. Express 6, 045101 (2013).

36) K. Uchida and S. Okada, Phys. Rev. B 76, 155436 (2007).

37) P. Hohenberg and W. Kohn, Phys. Rev. 136, B864 (1964).

38) W. Kohn and L. J. Sham, Phys. Rev. 140, A1133 (1965).

39) Y. Morikawa, K. Iwata, and K. Terakura, Appl. Surf. Sci. 169-170, 11 (2000).

40) J. P. Perdew and A. Zunger, Phys. Rev. B 23, 5048 (1981).

41) D. M. Ceperley and B. J. Alder, Phys. Rev. Lett. 45, 566 (1980).

42) D. Vanderbilt, Phys. Rev. B 41, 7892 (1990).

43) M. Otani and O. Sugino, Phys. Rev. B 73, 115407 (2006). 